PROCEEDINGS OF THE

AMERICAN MATHEMATICAL SOCIETY

Volume 138, Number 2, February 2010, Pages 641-653

S 0002-9939(09)10094-1

Article electronically published on October 5, 2009

\title{
GLOBAL ESTIMATES IN ORLICZ SPACES FOR THE GRADIENT OF SOLUTIONS TO PARABOLIC SYSTEMS
}

\author{
SUN-SIG BYUN AND SEUNGJIN RYU
}

(Communicated by Tatiana Toro)

\begin{abstract}
We find not only an optimal regularity requirement on the coefficients, but also a lowest level of regularity on the boundary for the global estimate of the gradient of a parabolic system in the setting of Orlicz spaces.
\end{abstract}

\section{INTRODUCTION}

Recently there have been active research activities on the regularity theory of the maximum order derivatives of the solutions of a certain class of partial differential equations and systems in the setting of Orlicz spaces; see [1, 5, 8, 23, 24, 25, 26, 27, etc. Most of these works have been achieved via an approach, the so-called maximal function free technique, which was first introduced by E. Acerbi and G. Mingione in the fine paper [2. This approach has merit in that it can completely avoid the use of any maximal function theory and it is suitable for situations in which scaling in time and space differs, such as $p$-Laplacian parabolic equations and systems; see 2]. However, as we have seen in most of the results, this approach does need an approximation argument to justify a priori estimates and reach the general case, which makes a complete proof relatively very technical and delicate.

In this paper we revisit a maximal function technique, which has been introduced in [9] and developed for the global estimates in the series of papers [4, 6, 7], to find a direct and elementary proof for some optimal regularity results in the setting of Orlicz spaces. We use here an approximation lemma which is based on higher integrability results on very nonsmooth domains and the smallness of the nonhomogeneous term (see Lemma 3.3), instead of weak compactness method as were used in previous work [4, 6, 7]. Needless to say, we do not need an approximation procedure based on a variable domain technique as was employed in [5, 8, 27.

This work is motivated for the purpose of finding a natural extension of the $L^{p}$ regularity, $1<p<\infty$, for the relevant differential operators from the fact that Orlicz spaces have been considered as one of the most natural generalizations of the

Received by the editors May 8, 2009.

2000 Mathematics Subject Classification. Primary 35K40, 35R05; Secondary 46E30, 46E35.

Key words and phrases. Gradient estimate, Orlicz space, parabolic system, maximal function, covering lemma, Reifenberg domain.

This work was supported by the Korea Research Foundation Grant funded by the Korean Government (KRF-2008-314-C00024).

(C)2009 American Mathematical Society Reverts to public domain 28 years from publication 
classical Lebesgue spaces. We refer to monographs [3, 13, 15, 20] for more details concerning Orlicz spaces.

In this paper we consider a second-order linear parabolic system in divergence form with possibly measurable coefficients in a very irregular domain to find a reasonable answer to what might be the weakest smoothness of the coefficients and the lowest level of regularity on the boundary for the global estimates in Orlicz spaces for the gradient of such a system. For second-order linear parabolic equations, the global $L^{p}$-regularity was obtained in the recent work [4] under a very weak assumption on the coefficients and a mild geometric condition on the boundary. The main point in 4 is that the boundary of the domain can be very rough; namely, it can be a Reifenberg flat domain whose geometric condition exhibits a very low level of regularity, prescribing that at all scales the boundary can be trapped between two hyperplanes, depending on the scale chosen. We refer to 6, 10, 21, 22, etc., for more details concerning Reifenberg domains. The assumption on the coefficients in [4] is that the coefficients can belong to small $B M O$ on the time slice while they are allowed to be measurable on the space slice. We refer to [5, 12, 14, etc., for a further discussion on such a type of class. In a similar spirit as in 4, the aim of this paper is not only to find a version of the result in 4 for systems, but also to extend the $L^{p}$-regularity of this kind to an appropriate regularity result in the setting of Orlicz spaces.

This paper is organized as follows. In Section 2 we introduce some notation and a problem under consideration, review Orlicz spaces, clarify regularity assumptions on the coefficients and boundary, and state the main result in Theorem 2.9. In Section 3 we discuss a useful setting under the present regularity assumptions, find an approximation lemma via a perturbation theory based on higher integrability results, and discuss a Vitali-type covering argument based on a maximal function operator. The last section is devoted to giving a complete proof of the main result in the setting of Orlicz spaces.

\section{Problem and main Result}

We start this section with some notation.

(1) The space and time variables are $(x, t)=\left(x^{\prime}, x_{n}, t\right)$ with their standard Lebesque measures $d x d t$. A typical point in $\mathbb{R}^{n} \times \mathbb{R}$ is $(y, s)$.

(2) $B_{\rho}=\left\{x \in \mathbb{R}^{n}:|x|<\rho\right\}$ is an open ball in $\mathbb{R}^{n}$ with center 0 and radius $\rho>0, B_{\rho}(y)=B_{\rho}+y, B_{\rho}^{+}=B_{\rho} \cap\left\{x: x_{n}>0\right\}, B_{\rho}^{+}(y)=B_{\rho}^{+}+y$, $T_{\rho}=B_{\rho} \cap\left\{x: x_{n}=0\right\}$, and $T_{\rho}(y)=T_{\rho}+y$.

(3) $\Omega_{\rho}=\Omega \cap B_{\rho}, \Omega_{\rho}(y)=\Omega \cap B_{\rho}(y)$.

(4) $\partial \Omega$ is the boundary of $\Omega, \partial_{w} \Omega_{\rho}=\partial \Omega \cap B_{\rho}$ is the wiggled part of $\partial \Omega_{\rho}$ and $\partial_{c} \Omega_{\rho}=\partial \Omega \cap\left\{x: x_{n}>0\right\}$ is the curved part of $\partial \Omega_{\rho}$.

(5) $Q_{\rho}=B_{\rho} \times\left(-\rho^{2}, \rho^{2}\right]$ is a centered parabolic cube, $Q_{\rho}(y, s)=Q_{\rho}+(y, s)$, $Q_{\rho}^{+}=B_{\rho}^{+} \times\left(-\rho^{2}, \rho^{2}\right]$ and $Q_{\rho}^{+}(y, s)=Q_{\rho}^{+}+(y, s)$.

(6) $S_{\rho}=T_{\rho} \times\left(-\rho^{2}, \rho^{2}\right], K_{\rho}=\Omega_{\rho} \times\left(-\rho^{2}, \rho^{2}\right]$ and $K_{\rho}(y, s)=K_{\rho}+(y, s)$.

(7) $\partial_{w} K_{\rho}=\partial_{w} \Omega_{\rho} \times\left(-\rho^{2}, \rho^{2}\right]$.

With the notation above we consider the following Dirichlet problem for a parabolic system in divergence form:

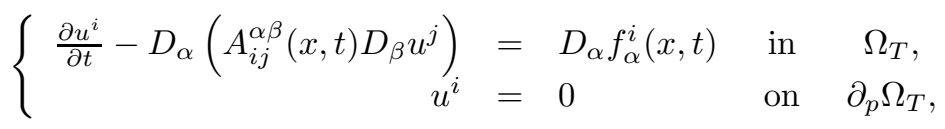


for $i=1, \ldots, m$ with $m>1$. Here $\Omega$ is a bounded domain in $\mathbb{R}^{n}$ with the nonsmooth boundary $\partial \Omega$ and $\Omega_{T}=\Omega \times(0, T]$ is a parabolic cylinder with the parabolic boundary $\partial_{p} \Omega_{T}=\partial \Omega \times[0, T] \cup \Omega \times\{t=0\}$ and $\mathbf{f}=\left\{f_{\alpha}^{i}\right\}$ is a given $m \times n$ matrix with $|\mathbf{f}|^{2} \in L^{\phi}\left(\Omega_{T}\right)$ for a given young function $\phi$ satisfying the $\triangle_{2} \cap \nabla_{2}$-condition; see Definitions 2.2 and 2.4. As usual, repeated indices mean summation; $\alpha, \beta$ are summed from 1 to $n$ and $i, j$ from 1 to $m$. Here and in the sequel we use the summation convention over repeated indices. We suppose that the tensor matrix $A_{i j}^{\alpha \beta}: \mathbb{R}^{n} \times \mathbb{R} \longrightarrow \mathbb{R}^{m^{2} n^{2}}$ is always assumed to be uniformly bounded, that is,

$$
\left|A_{i j}^{\alpha \beta}(x, t)\right| \leq L
$$

and satisfies the uniform parabolicity condition; that is,

$$
A_{i j}^{\alpha \beta}(x, t) \xi_{\alpha}^{i} \xi_{\beta}^{j} \geq \theta|\xi|^{2}
$$

for almost every $(x, t) \in \mathbb{R}^{n} \times \mathbb{R}$, for all $m \times n$ matrices $\xi=\left\{\xi_{\alpha}^{i}\right\}$ and for some positive constants $\theta, L$.

We will start with a classical definition of weak solutions.

Definition 2.1. We say that

$$
u=\left(u^{1}, \ldots, u^{m}\right) \in C^{0}\left(0, T ; L^{2}\left(\Omega, \mathbb{R}^{m}\right)\right) \cap L^{2}\left(0, T ; H_{0}^{1}\left(\Omega, \mathbb{R}^{m}\right)\right)
$$

is a weak solution of (2.1) if we have the following weak integral formulation:

$$
\int_{\Omega_{T}} u^{i} \varphi_{t}^{i}-A_{i j}^{\alpha \beta} D_{\beta} u^{j} D_{\alpha} \varphi^{i} d x d t=\int_{\Omega_{T}} f_{\alpha}^{i} D_{\alpha} \varphi^{i} d x d t
$$

for every test function

$$
\varphi=\left(\varphi^{1}, \ldots, \varphi^{m}\right) \in H^{1}\left(0, T ; L^{2}\left(\Omega, \mathbb{R}^{m}\right)\right) \cap L^{2}\left(0, T ; H_{0}^{1}\left(\Omega, \mathbb{R}^{m}\right)\right)
$$

with $\varphi=0$ for $t=0$ and $t=T$.

It is well known that the Dirichlet problem (2.1) has a unique weak solution $u$ with the regularity

$$
u \in H^{\frac{1}{2}}\left(0, T ; L^{2}\left(\Omega, \mathbb{R}^{m}\right)\right) \cap L^{2}\left(0, T ; H_{0}^{1}\left(\Omega, \mathbb{R}^{m}\right)\right)
$$

with the estimate

$$
\left\||D u|^{2}\right\|_{L^{1}\left(\Omega_{T}\right)} \leq c\left(1+\left\||\mathbf{f}|^{2}\right\|_{L^{1}\left(\Omega_{T}\right)}\right)
$$

for some constant $c=c\left(\theta, L,\left|\Omega_{T}\right|\right)$, provided that $|\mathbf{f}|^{2} \in L^{1}\left(\Omega_{T}\right)$ and $\Omega$ is bounded.

An Orlicz space is an appropriate substitute for an $L^{1}$-space when the $L^{1}$-space does not work. Here we are investigating how the Orlicz-regularity of $|\mathbf{f}|^{2}$ is reflected to the Orlicz-regularity of $|D u|^{2}$. To be more specific let us give some definitions and preliminary lemmas on the general Orlicz-Sobolev spaces. A nonnegative realvalued function $\phi$ defined on $[0, \infty)$ is called a Young function if it is increasing, convex and satisfies

$$
\phi(0)=0 ; \phi(\infty)=\lim _{\rho \rightarrow \infty} \phi(\rho)=\infty ; \lim _{\rho \rightarrow 0} \frac{\phi(\rho)}{\rho}=0 ; \lim _{\rho \rightarrow \infty} \frac{\phi(\rho)}{\rho}=\infty .
$$

Given a Young function $\phi$ and a bounded domain $U \in \mathbb{R}^{n} \times \mathbb{R}$, the Orlicz class $K^{\phi}(U)$ is the set of all measurable functions $v: U \rightarrow \mathbb{R}$ satisfying

$$
\int_{U} \phi(|v|) d x d t<\infty
$$


The Orlicz space $L^{\phi}(\Omega)$ is the smallest linear space under pointwise addition and scalar multiplication containing $K^{\phi}(U)$. Note that the Orlicz class $K^{\phi}(U)$ is in general just a convex set and not a linear space. But if $\phi$ satisfies the following so-called $\Delta_{2}$-condition, then the Orlicz class $K^{\phi}(U)$ is always a linear space and coincides with the Orlicz space $L^{\phi}(U)$ as a linear space.

Definition 2.2. A Young function $\phi$ is said to satisfy the $\Delta_{2}$-condition, denoted by $\phi \in \Delta_{2}$, if for some number $\kappa>1$,

$$
\phi(2 \rho) \leq \kappa \phi(\rho) \quad \forall \rho \geq 0 .
$$

This is the case if and only if for every $r>1$ there exists a positive constant $\kappa_{1}=\kappa_{1}(r)$ such that for all $\rho \geq 0, \phi(r \rho) \leq \kappa_{1} \phi(\rho)$.

Remark 2.3. Given the Young function $\phi \in \Delta_{2}$, the Luxemburg norm $\|\cdot\|_{L^{\phi}(U)}$ is defined as

$$
\|v\|_{L^{\phi}(U)}=\inf \left\{\rho>0: \int_{U} \phi(|v| / \rho) d x d t \leq 1\right\} .
$$

Note that the space $\left(L^{\phi}(U),\|\cdot\|_{L^{\phi}(U)}\right)$ is a Banach space with $C_{0}^{\infty}(U)$ dense in the Banach space $L^{\phi}(U)$.

Now let us introduce the main condition on $\phi$, the so-called $\nabla_{2}$-condition.

Definition 2.4. A Young function $\phi$ is said to satisfy the $\nabla_{2}$-condition, denoted by $\phi \in \nabla_{2}$, if for some number $\rho_{0}>1$,

$$
2 \rho_{0} \leq \frac{\phi\left(\rho_{0} \rho\right)}{\phi(\rho)}, \quad \forall \rho>0
$$

Here and in the sequel, a given Young function is always assumed to satisfy both $\Delta_{2}$ and $\nabla_{2}$ conditions, denoted by $\phi \in \Delta_{2} \cap \nabla_{2}$. These doubling-type conditions ensure that a Young function grows neither too slowly nor too fast and they are unavoidable for the type of regularity under consideration (see [23]), and they ensure that a Young function grows neither too slowly nor too fast.

Remark 2.5. The function $\phi(\rho)=\rho^{p}, p>1$, satisfies the $\Delta_{2} \cap \nabla_{2}$ condition. Thus the Lebesque spaces $L^{p}$ with $1<p<\infty$ are special cases of Orlicz spaces $L^{\phi}$ in this literature.

An important relationship between Lebesgue spaces and the Orlicz spaces considered here is stated below.

Lemma 2.6. Let $\phi$ be a Young function satisfying the $\Delta_{2} \cap \nabla_{2}$ condition. Then for some $\rho_{2} \geq \rho_{1}>1$,

$$
L^{\rho_{2}}(U) \subset L^{\phi}(U) \subset L^{\rho_{1}}(U) \subset L^{1}(U) .
$$

Now we return to the Dirichlet problem (2.1). Given a Young function $\phi \in$ $\Delta_{2} \cap \nabla_{2}$, if $|\mathbf{f}|^{2}$ belongs to $L^{\phi}\left(\Omega_{T}\right)$, then (2.8) in Lemma 2.6 implies that $\mathbf{f} \in$ $L^{2}\left(\Omega_{T} ; \mathbb{R}^{m n}\right)$. Thus it follows from (2.4) that the Dirichlet problem (2.1) has a unique weak solution $u$ satisfying $|\nabla u|^{2} \in L^{1}\left(\Omega_{T}\right)$. But it is generally not true that $|\nabla u|^{2} \in L^{\phi}\left(\Omega_{T}\right)$ under the basic assumptions that $\Omega$ is bounded and the $A_{i j}^{\alpha \beta}$ satisfy (2.2) and (2.3). Obviously, such a regularity requires some additional smoothness of the coefficients as well as some additional regularity of the boundary. In this 
paper we want to ask what is the weakest condition to place on $A_{i j}^{\alpha \beta}$ besides (2.2) and (2.3) and what is the lowest level of geometric structure on $\partial \Omega$ under which

$$
|\mathbf{f}|^{2} \in L^{\phi}\left(\Omega_{T}\right) \Longrightarrow|\nabla u|^{2} \in L^{\phi}\left(\Omega_{T}\right)
$$

for a given Young function $\phi \in \Delta_{2} \cap \nabla_{2}$. Our main purpose in this paper is to prove (2.9) provided the $A_{i j}^{\alpha \beta}$ have small BMO semi-norms of weak type (see Definition 2.7) and $\partial \Omega$ is sufficiently flat in the Reifenberg sense (see Definition 2.8).

To introduce the main result, we first recall the notation mentioned earlier in this section and introduce some more. The integral average of an integrable function $f$ on a bounded subset $U$ of $\mathbb{R}^{n} \times \mathbb{R}$ is defined by

$$
\bar{f}_{U}=f_{U} f(x, t) d x d t=\frac{1}{|U|} \int_{U} f(x, t) d x d t .
$$

For each fixed $t \in \mathbb{R}$ and for each bounded subset $E$ of $\mathbb{R}^{n}$, we also denote by $\bar{f}_{E}(t)$ the integral average of $f(\cdot, t)$ on $E$, namely,

$$
\bar{f}_{E}(t)=f_{E} f(x, t) d x=\frac{1}{|E|} \int_{E} f(x, t) d x .
$$

Here and in the sequel $\delta>0$ is a small universal constant, to be determined later. This number is invariant under a scaling and normalization for the problem (2.1). The number $R$ can be any other constant, such as 1 , or any other constant, such as 48 later in this paper, by scaling the PDE in (2.1).

Low regularity of the coefficients under consideration means that the $A_{i j}^{\alpha \beta}$ are in BMO space with respect to the space variable and are merely measurable with respect to the time variable previously used in [5, 12. More precisely we have the following assumption.

Definition 2.7. We say that the $A_{i j}^{\alpha \beta}$ are weakly $(\delta, R)$-vanishing if

$$
\sup _{Q_{r}(y, s)} \sqrt{f_{Q_{r}(y, s)}\left|A_{i j}^{\alpha \beta}(x, t)-\overline{A_{i j}^{\alpha \beta} B_{r}(y)}(t)\right|^{2} d x d t} \leq \delta,
$$

where $0<r \leq R$ and $(y, s) \in \mathbb{R}^{n+1}$.

The class considered in this paper, that is, the class of functions satisfying the weak small BMO condition (2.11), is much larger than the class of functions satisfying the classic VMO condition or strong small BMO condition previously used in [6, 7, 8, 16, 17. For example, one takes $f(x, t)=g(x) h(t)$ such that the BMO semi-norm of $g(x)$ is small enough to keep $f(x, t)$ satisfying the weak small BMO condition, though $h(t)$ is allowed to have big jumps at a finite number of times. Of course such functions do not satisfy the strong small BMO condition. This class also includes all the measurable functions of the time variable.

As mentioned in the Introduction, the regularity of the boundary is the Reifenberg flatness of a set. Our geometric condition on the boundary of a domain is stated as follows.

Definition 2.8. We say that $\Omega$ is $(\delta, R)$-Reifenberg flat if for every point $x \in \partial \Omega$ and every scale $r \in(0, R]$ there exists a coordinate system, which we still denote by $\left\{x_{1}, \ldots, x_{n}\right\}$, so that in this new coordinate system,

$$
B_{r} \cap\left\{x_{n}>\delta r\right\} \subset B_{r} \cap \Omega \subset B_{r} \cap\left\{x_{n}>-\delta r\right\} .
$$


A Reifenberg flat set was first introduced by Reifenberg in the paper [21. This set is locally a topological disk if $\delta$ is sufficiently small; see [10,21]. It turns out that a Reifenberg flat domain exhibits minimal geometric properties for some natural properties in geometric analysis to hold; see [11. A good example of Reifenberg flat domains considered here is a flat version of the well-known Van Koch snowflake when the angle of the spike with respect to the horizontal is sufficiently small. A Reifenberg flat domain might have a fractal boundary that is like a coastline, a crystal grain boundary, or atomic clusters. We remark that an interior $\epsilon$-neighborhood of a $(\delta, R)$-Reifenberg flat domain is a Lipschitz domain for small $\epsilon>0$ provided $\delta>0$ is so small that the domain is a $W^{1, p}$ extension domain; see [6]. On the other hand a Lipschitz domain is $(\delta, R)$-Reifenberg flat provided its Lipschitz constants are small enough; see [22].

Let us state the main results of this paper.

Theorem 2.9. Let $\phi$ be a Young function satisfying $\phi \in \Delta_{2} \cap \nabla_{2}$. Then there exists a small positive $\delta=\delta\left(\theta, L, n, m, \phi,\left|\Omega_{T}\right| /\left|Q_{1}\right|\right)$ and a positive constant $c=$ $\left(\theta, L, n, m, \phi,\left|\Omega_{T}\right|\right)$ such that if the $A_{i j}^{\alpha \beta}$ are weakly $(\delta, R)$-vanishing, $\Omega$ is $(\delta, R)$ Reifenberg flat and $|\mathbf{f}|^{2} \in L^{\phi}\left(\Omega_{T}\right)$, then the unique weak solution u satisfies $|D u|^{2} \in$ $L^{\phi}\left(\Omega_{T}\right)$ with the estimate

$$
\int_{\Omega_{T}} \phi\left(|D u|^{2}\right) d x d t \leq c\left(1+\int_{\Omega_{T}} \phi\left(\left|\mathbf{f}^{2}\right|\right) d x d t\right) .
$$

As a corollary of Theorem 2.9, we have the following $W^{1, p}$-regularity for each $1<p<\infty$.

Corollary 2.10. Let $1<p<\infty$. Then there exists a small positive constant $\delta=\delta\left(\theta, L, n, m, p,\left|\Omega_{T}\right| /\left|Q_{1}\right|\right)$ and a positive constant $c=\left(\theta, L, n, m, p,\left|\Omega_{T}\right|\right)$

such that if the $A_{i j}^{\alpha \beta}$ are weakly $(\delta, R)$-vanishing, $\Omega$ is $(\delta, R)$-Reifenberg flat and $\mathbf{f} \in L^{p}\left(\Omega_{T}, \mathbb{R}^{m n}\right)$, then the unique weak solution u satisfies $|D u| \in L^{p}\left(\Omega_{T}\right)$ with the estimate

$$
\int_{\Omega_{T}}|D u|^{p} d x d t \leq c\left(1+\int_{\Omega_{T}}|\mathbf{f}|^{p} d x d t\right) .
$$

Proof. If $p>2$, then we take $\phi(\rho)=\rho^{\frac{p}{2}}$ and apply Theorem 2.9 to find the corollary. The case $p=2$ is classical, and the case $1<p<2$ can be recovered by duality.

\section{MAXIMAL FUNCTION APPROACH}

For the sake of convenience and clarity, we employ the letter $c$ through this section to denote any constant which can be explicitly computed in terms of known quantities such as $\theta, L, n, m, \phi$ and the geometry of related domains. Thus the exact value denoted by $c$ may change from line to line in a given computation. We also point out that our weak solutions $u \in C^{0}\left(0, T ; L^{2}\left(\Omega, \mathbb{R}^{m}\right)\right) \cap L^{2}\left(0, T ; H_{0}^{1}\left(\Omega ; \mathbb{R}^{m}\right)\right)$ of (2.1) will be hereafter assumed to be defined on $\Omega \times \mathbb{R}$ for the following reasons. The solution $u$ and the system can be extended forward by taking $\mathbf{f}=0$ so that all properties in question are preserved. For a backward extension, one can use the zero extension of $u$. Along with the observation above, because the idea of the interior estimates in this context is essentially the same as that of boundary estimates, we treat only the discussion of the estimates on the lateral boundary. Our object in this section is twofold. Initially, we find an approximation lemma; 
see Lemma 3.3. We then obtain the decay of the measure of some level set for the gradient; see Lemma 3.6. For simplicity, we take $R=6$ for Lemma 3.3. Namely, we assume

$$
\left\{(x, t) \in Q_{6}: x_{n}>0\right\} \subset K_{6} \subset\left\{(x, t) \in Q_{r}: x_{n}>-12 \delta r\right\}
$$

and

$$
f_{Q_{6}^{+}}\left|A_{i j}^{\alpha \beta}(x, t)-\overline{A_{i j}^{\alpha \beta} B_{6}^{+}}(t)\right|^{2} d x d t \leq \delta^{2} .
$$

With these assumptions we consider a corresponding localized problem

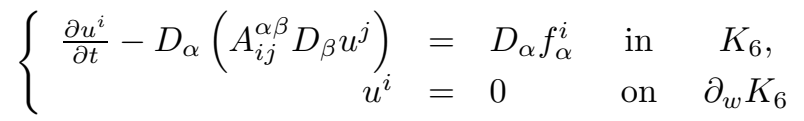

and its homogeneous problem

$$
\left\{\begin{array}{rlllr}
\frac{\partial w^{i}}{\partial t}-D_{\alpha}\left(A_{i j}^{\alpha \beta} D_{\beta} w^{j}\right) & = & 0 & \text { in } & K_{6} \\
w^{i} & = & u^{i} & \text { on } & \partial_{p} K_{6}
\end{array}\right.
$$

where $u$ is a weak solution under consideration. Plus we also consider a limiting problem

$$
\left\{\begin{array}{rllll}
\frac{\partial v^{i}}{\partial t}-D_{\alpha}\left(\overline{A_{i j}^{\alpha \beta} B_{5}^{+}}(t) D_{\beta} v^{j}\right) & =0 & \text { in } & Q_{5}^{+}, \\
v^{i} & = & 0 & \text { on } & S_{5} .
\end{array}\right.
$$

The following higher integrability result near a Reifenberg flat domain follows from the recent papers [18, 19] by Mikko Parviainen in which higher integrability results were discussed near a boundary which satisfies a very mild condition, the so-called capacity density condition, which is weaker than our Reifenberg flatness condition.

Lemma 3.1. Let $w$ be a weak solution of (3.4) with the assumptions (2.2), (2.3), (3.1), (3.2) and

$$
f_{K_{6}}|\nabla u|^{2} d x d t \leq 1 .
$$

Then $|\nabla w| \in L^{2+\epsilon_{0}}\left(K_{5}\right)$ with the estimate

$$
f_{K_{5}}|\nabla w|^{2+\epsilon_{0}} d x d t \leq c
$$

for some positive constants $\epsilon_{0}=\epsilon_{0}(n, m, \theta, L, \delta)$.

Now we observe that if $v$ is a weak solution of (3.5), then by taking tangential derivatives to this equation, we see that for each $1 \leq k \leq n, D_{k} v$ is a weak solution of

$$
\left\{\begin{array}{rllll}
\frac{\partial\left(D_{k} v\right)^{i}}{\partial t}-D_{\alpha}\left(\overline{A_{i j}^{\alpha \beta}{ }_{5}^{+}}(t) D_{\beta}\left(D_{k} v\right)^{j}\right) & =0 & \text { in } & Q_{5}^{+}, \\
\left(D_{k} v\right)^{i} & = & 0 & \text { on } & S_{5} .
\end{array}\right.
$$

Then by this observation and from standard $L^{\infty}$-regularity for (3.7), we have the following Lipschitz regularity up to the flat boundary.

Lemma 3.2. For any weak solution $v$ of (3.5) with (2.2), (2.3) and (3.2), we have

$$
\|\nabla v\|_{L^{\infty}\left(Q_{4}^{+}\right)}^{2} \leq c f_{Q_{5}^{+}}|\nabla v|^{2} d x d t
$$


Now we are all set up to prove the following approximation lemma.

Lemma 3.3. For $0<\epsilon<1$ fixed, there exists a small $\delta=\delta(\epsilon)>0$ such that for any weak solution $u$ of (3.3) with the assumptions (2.2), (2.3), (3.1), (3.2) and

$$
f_{K_{6}}|\nabla u|^{2} d x d t \leq 1, f_{K_{6}}|\mathbf{f}|^{2} d x d t \leq \delta^{2},
$$

there exists a weak solution $v$ of (3.5) such that

$$
f_{K_{4}}|\nabla(u-v)|^{2} d x d t \leq \epsilon^{2},
$$

where $\bar{v}$ is the zero extension of $v$ from $Q_{5}^{+}$to $K_{5}$.

Proof. Let $w$ be a weak solution of (3.4). Then a direct computation shows that $u-w$ is the weak solution of

$$
\left\{\begin{array}{rlrl}
\frac{\partial(u-w)^{i}}{\partial t}-D_{\alpha}\left(A_{i j}^{\alpha \beta} D_{\beta}(u-w)^{j}\right) & =D_{\alpha} f_{\alpha}^{i} & \text { in } \quad K_{6}, \\
(u-w)^{i} & =0 & & \text { on } \partial_{p} K_{6},
\end{array}\right.
$$

and we see from the standard $L^{2}$-estimate and (3.8) that

$$
f_{K_{6}}|\nabla(u-w)|^{2} d x d t \leq c f_{K_{6}}|\mathbf{f}|^{2} d x d t \leq c \delta^{2} .
$$

Now according to Lemma 3.1 we see that $|\nabla w|$ is in $L^{2+\epsilon_{0}}\left(K_{5}\right)$ with the estimate

$$
f_{K_{5}}|\nabla w|^{2+\epsilon_{0}} d x d t \leq c
$$

for some universal constant $\epsilon_{0}$. We next let $h$ be the weak solution of

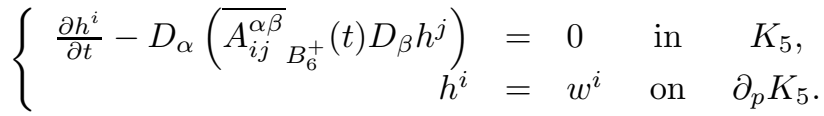

Then from (3.4) it follows that $\eta:=w-h$ is the weak solution of

$$
\left\{\begin{aligned}
\frac{\partial \eta^{i}}{\partial t}-D_{\alpha}\left(\overline{A_{i j}^{\alpha \beta} B_{6}^{+}}(t) D_{\beta} \eta^{j}\right) & =D_{\alpha}\left(\left[A_{i j}^{\alpha \beta}-\overline{A_{i j}^{\alpha \beta} B_{6}^{+}}(t)\right] D_{\beta} w^{j}\right) & & \text { in } K_{5}, \\
\eta^{i} & =0 & & \text { on } \partial_{p} K_{5} .
\end{aligned}\right.
$$

Thus we calculate using Hölder's inequality and (3.10) that

$$
f_{K_{5}}|\nabla(w-h)|^{2} d x d t \leq c\left(f_{K_{5}}\left|A_{i j}^{\alpha \beta}-\overline{A_{i j}^{\alpha \beta}} B_{6}^{+}(t)\right|^{\frac{2\left(\epsilon_{0}+2\right)}{\epsilon_{0}}} d x d t\right)^{\frac{\epsilon_{0}}{\epsilon_{0}+2}} .
$$

Then from Hölder's inequality, (2.2) and (3.2) we see that

$$
f_{K_{5}}|\nabla(w-h)|^{2} d x d t \leq c \delta^{\sigma}
$$

for some positive number $\sigma=\sigma(m, n, \theta, L)$. Now we select a smooth cutoff function $\phi=\phi\left(x_{n}\right)$ such that

$$
\phi=1 \text { if } x_{n} \geq 1, \phi=0 \text { if } x_{n} \leq 0,\left|\phi^{\prime}\right| \leq c \text { and } 0 \leq \phi \leq 1 .
$$

We next let $v$ be the weak solution of

$$
\left\{\begin{array}{rlllr}
\frac{\partial v^{i}}{\partial t}-D_{\alpha}\left(\overline{A_{i j}^{\alpha \beta}}{ }_{B_{6}^{+}}(t) D_{\beta} v^{j}\right)_{v^{i}} & = & 0 & \text { in } & h_{5}^{+} \phi \\
& \text { on } & \partial_{p} Q_{5}^{+} .
\end{array}\right.
$$


Then it follows from (3.1) and (3.13) that $v=0$ on $S_{5}$, so by Lemma 3.2 and (3.8), we have

$$
\|\nabla v\|_{L^{\infty}\left(Q_{4}^{+}\right)}^{2} \leq c f_{Q_{5}^{+}}|\nabla v|^{2} d x d t \leq c f_{K_{6}}|\nabla u|^{2} d x d t \leq c .
$$

We let $\bar{v}$ be the zero extension of $v$ from $Q_{5}^{+}$to $K_{5}$. Then by a direct computation one can see that $\bar{v}$ is a weak solution of

$$
\left\{\begin{array}{rlll}
\frac{\partial \bar{v}^{i}}{\partial t}-D_{\alpha}\left(\overline{A_{i j}^{\alpha \beta} B_{6}^{+}}(t) D_{\beta} \bar{v}^{j}\right) & =D_{n} g_{n}^{i} & & \text { in } \quad K_{5}, \\
\bar{v}^{i} & =0 & & \text { on } \partial_{w} K_{5},
\end{array}\right.
$$

where

$$
g_{n}^{i}(x, t)= \begin{cases}0 & \text { if }(x, t) \in Q_{5}^{+}, \\ \bar{A}_{i j B_{6}^{+n}}^{n n}(t) D_{n} v^{j}\left(x^{\prime}, 0, t\right) & \text { if }(x, t) \in K_{5} \backslash Q_{5}^{+} .\end{cases}
$$

Then from (3.11) and (3.16), we find that $h-\bar{v}$ is a weak solution of

$$
\left\{\begin{array}{rlrl}
\frac{\partial(h-\bar{v})^{i}}{\partial t}-D_{\alpha}\left(\overline{A_{i j}^{\alpha \beta} B_{5}^{+}}(t) D_{\beta}(h-\bar{v})^{j}\right) & =-D_{n} g_{n}^{i} & & \text { in } \\
(h-\bar{v})^{i} & =0 & & K_{5}, \\
& & \text { on } \partial_{w} K_{5} .
\end{array}\right.
$$

Now from (3.17) and by a standard $L^{2}$-estimate for (3.19), we have

$$
f_{K_{4}}|\nabla(h-\bar{v})|^{2} d x d t \leq \int_{K_{4}}\left|g_{n}^{i}\right|^{2} d x d t \leq c \delta
$$

where the last inequality comes from (2.2), (3.1) and (3.15).

Therefore, it follows from (3.9), (3.12) and (3.19) that

$$
f_{K_{4}}|\nabla(u-\bar{v})|^{2} d x d t \leq c\left(\delta^{2}+\delta^{\sigma}+\delta\right) .
$$

Now the conclusion directly comes from this estimate (3.20) and by taking $\delta>0$ so that

$$
c\left(\delta+\delta^{\sigma}+\delta\right)=\epsilon
$$

This completes the proof.

Our technique is based on the maximal function operator.

Definition 3.4. Given a function $f$ defined on $\mathbb{R}^{n} \times \mathbb{R}$, the Hardy-Littlewood maximal function $\mathcal{M} f$ of $f$ is a function such that

$$
(\mathcal{M} f)(x, t)=\sup _{Q_{r}(x, t)} f_{Q}|f(y, s)| d y d s
$$

where the supremum is taken over parabolic cylinders $Q_{r}(x, t)$ in $\mathbb{R}^{n} \times \mathbb{R}$ which are centered at the point $(y, s)$ of size $r>0$. If $f$ is defined only in a bounded domain $U \subset \mathbb{R}^{n} \times \mathbb{R}$, we define its restricted maximal function as

$$
\mathcal{M}_{U} f=\mathcal{M}\left(f \chi_{U}\right)
$$

where $\chi$ is the standard characteristic function on $U$.

Similarly to the proof of Lemma 8 in [7, using the maximal function operator and the approximation Lemma 3.3, we get 
Lemma 3.5. There is a universal constant $n_{1}>1$ so that for $0<\epsilon<1$ fixed, there exists a small $\delta=\delta(\epsilon)>0$ such that if $u$ is a weak solution of (2.1) under the assumptions (2.2), (2.3), (3.1) and (3.2) with 6 replaced by 8 , and

$$
\left\{(x, t) \in K_{1}: \mathcal{M}\left(|\nabla u|^{2}\right) \leq 1\right\} \cap\left\{(x, t) \in K_{1}: \mathcal{M}\left(|\mathbf{f}|^{2}\right) \leq \delta^{2}\right\} \neq \emptyset,
$$

then

$$
\left|\left\{(x, t) \in K_{1}: \mathcal{M}\left(|\nabla u|^{2}\right)>n_{1}^{2}\right\} \cap Q_{1}\right|<\epsilon\left|Q_{1}\right| .
$$

Let us next fix $\epsilon$ and take $\delta$ and $n_{1}$ as given in Lemma 3.5. Then in view of a scaling invariance form of Lemma 3.5 and with a careful choice of cubes in the Vitali covering lemma, one can show the decay of the measure of the set $\{(x, t) \in$ $\left.\Omega_{T}: \mathcal{M}\left(|D u|^{2}\right)>n_{1}^{2}\right\}$. For a complete procedure to derive this decay, we refer to the early work in [4, 7].

Lemma 3.6. Let $u$ be a weak solution of (2.1). Assume that the $A_{i j}^{\alpha \beta}$ are weakly $(\delta, 48)$-vanishing and that $\Omega$ is $(\delta, 48)$-Reifenberg flat. Assume further that

$$
\left|\left\{(x, t) \in \Omega_{T}: \mathcal{M}\left(|\nabla u|^{2}\right)>n_{1}^{2}\right\}\right| \leq \epsilon\left|Q_{1}\right| .
$$

Then for each natural number $k \geq 1$, we have

$$
\begin{aligned}
\left|\left\{(x, t) \in \Omega_{T}: \mathcal{M}\left(|\nabla u|^{2}\right)>n_{1}^{2 k}\right\}\right| & \leq \epsilon_{1}^{k}\left|\left\{(x, t) \in \Omega_{T}: \mathcal{M}\left(|\nabla u|^{2}\right)>1\right\}\right| \\
& +\sum_{i=1}^{k} \epsilon_{1}^{i}\left|\left\{(x, t) \in \Omega_{T}: \mathcal{M}\left(|\mathbf{f}|^{2}\right)>\delta^{2} n_{1}^{2(k-i)}\right\}\right|,
\end{aligned}
$$

where $\epsilon_{1}=[10 /(1-\delta)]^{n+2} \epsilon$.

\section{Proof of the main Result}

We will use the following standard lemmas in classical measure theory on Orlicz spaces.

Lemma 4.1 ([13]). Let $\phi$ be a Young function $\phi \in \Delta_{2} \cap \nabla_{2}$ and $U$ a bounded domain in $\mathbb{R}^{n} \times \mathbb{R}$. If $f \in L^{\phi}(U)$, then $\mathcal{M} f \in L^{\phi}(U)$ and

$$
\int_{U} \phi(|f|) d x d t \leq \int_{U} \phi(\mathcal{M}(|f|)) d x d t \leq c \int_{U} \phi(|f|) d x d t
$$

with a constant $c$ independent of $f$.

Lemma 4.2 (13). Assume that $f$ is a nonnegative and measurable function in $\mathbb{R}^{n} \times \mathbb{R}$. Assume further that $f$ has a compact support in a bounded subset $U$ of $\mathbb{R}^{n} \times \mathbb{R}$. Let $\nu>0$ and $\sigma>1$ be constants. Then for any Young function $\phi \in \Delta_{2} \cap \nabla_{2}$, we have

$$
f \in L^{\phi}(U) \Longleftrightarrow S=\sum_{k \geq 1} \phi\left(\sigma^{k}\right)\left|\left\{(x, t) \in U: f(x, t)>\nu \sigma^{k}\right\}\right|<\infty
$$

and

$$
\frac{1}{c} S \leq \int_{U} \phi(|f|) d x d t \leq c(|U|+S),
$$

where $c>0$ is a constant depending only on $\nu, \sigma$, and $\phi$.

We are now in a position to give a complete proof of our main result, Theorem 2.9. 
Proof. We first assume $R=48$ by a scaling. We also assume

$$
\left|\left\{(x, t) \in \Omega_{T}: \mathcal{M}\left(|\nabla u|^{2}\right)>n_{1}^{2}\right\}\right|<\epsilon\left|Q_{1}\right|
$$

by replacing $u$ and $\mathbf{f}$ by $\tau u$ and $\tau \mathbf{f}$, respectively, for some small $\tau=\tau(\epsilon)>0$. Then from Lemma 3.6 we calculate

$$
\begin{aligned}
& \sum_{k=1}^{\infty} \phi\left(n_{1}^{2 k}\right)\left|\left\{(x, t) \in \Omega_{T}: \mathcal{M}\left(|\nabla u|^{2}\right)>n_{1}^{2 k}\right\}\right| \\
& \leq \sum_{k=1}^{\infty} \phi\left(n_{1}^{2 k}\right) \epsilon_{1}^{k}\left|\left\{(x, t) \in \Omega_{T}: \mathcal{M}\left(|\nabla u|^{2}\right)>1\right\}\right| \\
& +\sum_{k=1}^{\infty} \phi\left(n_{1}^{2 k}\right) \sum_{i=1}^{k} \epsilon_{1}^{i}\left|\left\{(x, t) \in \Omega_{T}: \mathcal{M}\left(|\mathbf{f}|^{2}\right)>\delta^{2} n_{1}^{2(k-i)}\right\}\right| \\
& =: S_{1}+S_{2} .
\end{aligned}
$$

Since $\phi \in \Delta_{2}$ and $n_{1}>1$, there exists $\kappa_{1}>1$ such that $\phi\left(n_{1}^{2}\right) \leq \kappa_{1} \phi(1)$; see Definition 2.2. By iteration, we see that $\phi\left(n_{1}^{2 k}\right) \leq \kappa_{1}^{k} \phi(1)$. Thus we find

$$
S_{1} \leq \phi(1)\left|\Omega_{T}\right| \sum_{k=1}^{\infty}\left(\kappa_{1} \epsilon_{1}\right)^{k} \leq c_{1} \sum_{k=1}^{\infty}\left(\kappa_{1} \epsilon_{1}\right)^{k}
$$

for some $c_{1}=c_{1}\left(\phi, \theta, L,\left|\Omega_{T}\right|, m, n\right)$. On the other hand, by using Fubini's theorem and by the same reasoning set for $S_{1}$, we have for each $i \geq 1$,

$$
S_{2} \leq \phi(1) \sum_{i=1}^{\infty}\left(\kappa_{1} \epsilon_{1}\right)^{i} \Sigma(i)
$$

where

$$
\Sigma(i)=\sum_{k=i}^{\infty} \phi\left(n_{1}^{2[k-i]}\right)\left|\left\{(x, t) \in \Omega_{T}: \mathcal{M}\left(|\mathbf{f}|^{2}\right)>\delta^{2} n_{1}^{2[k-i]}\right\}\right| .
$$

But then by Lemmas 4.1 and 4.2 , we have

$$
\Sigma(i) \leq c_{2} \int_{\Omega_{T}} \phi\left(|\mathbf{f}|^{2}\right) d x d t
$$

for some constant $c_{2}=c_{2}\left(\phi, \theta, L, \delta, m, n,\left|\Omega_{T}\right|\right)$. Thus from (4.4) we obtain

$$
S_{2} \leq c_{2}\left(\int_{\Omega_{T}} \phi\left(|\mathbf{f}|^{2}\right) d x d t\right) \sum_{k=1}^{\infty}\left(\kappa_{1} \epsilon_{1}\right)^{k} .
$$

We select $\delta$ so small that $\kappa_{1} \epsilon_{1}<1$ and use (4.3) and (4.5) to conclude that

$$
\sum_{k=1}^{\infty} \phi\left(n_{1}^{2 k}\right)\left|\left\{(x, t) \in \Omega_{T}: \mathcal{M}\left(|\nabla u|^{2}\right)>n_{1}^{2 k}\right\}\right| \leq c_{3}\left(1+\int_{\Omega_{T}} \phi\left(|\mathbf{f}|^{2}\right) d x d t\right)
$$

for some constant $c_{3}=c_{3}\left(\phi, \theta, L, m, n,\left|\Omega_{T}\right|\right)$. Now the conclusion follows from Lemmas 4.1 and 4.2 . This completes the proof. 


\section{REFERENCES}

1. E. Acerbi and G. Mingione, Gradient estimates for the $p(x)$-Laplacean system, J. Reine Angew. Math., 584 (2005), 117-148. MR2155087(2006f:35068)

2. E. Acerbi and G. Mingione, Gradient estimates for a class of parabolic systems, Duke Math. J., 136 (2) (2007), 285-320. MR2286632 (2007k:35211)

3. R. Adams, Sobolev spaces, Academic Press, New York, 1975. MR0450957 (56:9247)

4. S. Byun, Optimal $W^{1, p}$ regularity theory for parabolic equations in divergence form, J. Evol. Equ., 7 (3) (2007), 415-428. MR22328932 (2008i:35108)

5. S. Byun, Hessian estimates in Orlicz spaces for fourth-order parabolic systems in nonsmooth domains, J. Differential Equations, 246 (9) (2009), 3518-3234. MR.2515166

6. S. Byun and L. Wang, Parabolic equations in time dependent Reifenberg domains, Adv. Math., 212 (2) (2007), 797-818. MR2329320 (2008h:35131)

7. S. Byun and L. Wang, $W^{1, p}$ regularity for the conormal derivative problem with parabolic BMO nonlinearity in Reinfenberg domains, Discrete Contin. Dyn. Syst., 20 (3) (2008), 617637. MR2373207

8. S. Byun, F. Yao and S. Zhou, Gradient estimates in Orlicz space for nonlinear elliptic equations, J. Funct. Anal., 255 (8) (2008), 1851-1873. MR2462578

9. Luis A. Caffarelli and I. Peral, On $W^{1, p}$ estimates for elliptic equations in divergence form, Comm. Pure Appl. Math., 51 (1998), no. 1, 1-21. MR1486629 (99c:35053)

10. G. Hong and L. Wang, A geometric approach to the topological disk theorem for Reifenberg, Pacific J. Math., 233 (2) (2007), 321-339. MR2366379 (2008m:49203)

11. P. W. Jones, Quasiconformal mappings and extendability of functions in Sobolev spaces, Acta Math., 147 (1981), 71-88. MR631089 (83i:30014)

12. D. Kim and N. V. Krylov, Elliptic differential equations with coefficients measurable with respect to one variable and VMO with respect to the others, SIAM J. Math. Anal., 39 (2) (2007), 489-506. MR2338417 (2008j:35031)

13. V. Kokilashvili and M. Krbec, Weighted inequalities in Lorentz and Orlicz spaces, World Scientific Publishing Co., Inc., River Edge, NJ (1991). MR 1156767 (93g:42013)

14. N. V. Krylov, Parabolic equations with VMO coefficients in Sobolev spaces with mixed norms, J. Funct. Anal., 250 (2) (2007), 521-558. MR2352490 (2008f:35164)

15. W. Orlicz, Uber eine gewisse Klasse von Raumen vom Typus B, Bull. Int. Acad. Polon. Sci. A, 8/9 (1932), 207-220.

16. D. Palagachev, Quasilinear elliptic equations with VMO coefficients, Trans. Amer. Math. Soc., 347 (1995), 2481-2493. MR.1308019 (95k:35083)

17. D. Palagachev and L. Softova, A priori estimates and precise regularity for parabolic systems with discontinuous data, Discrete Contin. Dyn. Syst., 13 (2005), 721-742. MR2153140 (2006c:35120)

18. M. Parviainen, Global higher integrability for parabolic quasiminimizers in nonsmooth domains, Calc. Var. Partial Differential Equations, 31 (1) (2008), 75-98. MR2342615 (2009c:49073)

19. M. Parviainen, Global gradient estimates for degenerate parabolic equations in nonsmooth domains, Ann. Mat. Pura Appl. (4), 188 (2) (2009), 333-358. MR.2491806

20. M. M. Rao and Z. D. Ren, Theory of Orlicz spaces, Monographs and Textbooks in Pure and Applied Mathematics, 146, Marcel Dekker, Inc., New York (1991). MR1113700 (92e:46059)

21. E. Reinfenberg, Solution of the Plateau Problem for $m$-dimensional surfaces of varying topological type, Acta Math. 104 (1960), 1-92. MR0114145 (22:4972)

22. T. Toro, Doubling and flatness: Geometry of measures, Notices Amer. Math. Soc., 44 (9) (1997), 1087-1094. MR1470167 (99d:28010)

23. L. Wang, F. Yao, S. Zhou and H. Jia, Optimal regularity for the Poisson equation, Proceedings Amer. Math. Soc., 137 (2009), 2037-2047. MR2480285

24. F. Yao, Regularity theory in Orlicz spaces for the parabolic polyharmonic equations, Arch. Math. (Basel), 90 (5) (2008), 429-439. MR2414246

25. F. Yao, H. Jia, L. Wang and S. Zhou, Regularity theory in Orlicz spaces for the Poisson and heat equations, Commun. Pure Appl. Anal., 7 (2) (2008), 407-416. MR2373223 (2009d:35023) 
26. F. Yao, Y. Sun and S. Zhou, Gradient estimates in Orlicz spaces for quasilinear elliptic equation, Nonlinear Anal., 69 (8) (2008), 2553-2565. MR2446351

27. F. Yao and S. Zhou, Linear second-order divergence equations in Lipschitz domains, J. Math. Anal. Appl., 344 (1) (2008), 491-503. MR.2416323 (2009e:35048)

Department of Mathematics, Seoul National University, Seoul 151-747, Republic of Korea

E-mail address: byun@snu.ac.kr

Department of Mathematics, Seoul National University, Seoul 151-747, Republic of KOREA

E-mail address: sjryu@math.snu.ac.kr 